\title{
Uncertain Risk Assessment of Knowledge Management: Based on Set Pair Analysis
}

\author{
Guibin Yang and Hongyu Gao \\ School of Economics and Management, Harbin Engineering University, Harbin 150001, China \\ Correspondence should be addressed to Guibin Yang; yangguibin1975@163.com
}

Received 17 February 2016; Accepted 19 June 2016

Academic Editor: Piotr Luszczek

Copyright ( 2016 G. Yang and H. Gao. This is an open access article distributed under the Creative Commons Attribution License, which permits unrestricted use, distribution, and reproduction in any medium, provided the original work is properly cited.

\begin{abstract}
Since the knowledge resource becomes an important part of enterprise resources, the knowledge management risks could significantly affect the enterprise operation efficiency. Controlling knowledge management risks is one of the enterprise management tasks; the managers and researchers focus on how to effectively evaluate the risks. This paper aims to solve this problem and puts forward an evaluation model of the uncertain risks of knowledge management. Based on set pair theory, a model is established to identify the knowledge management risk. Through the concept of connection number, knowledge management risk is divided into five levels. Meanwhile, the development trend of knowledge management risk is classified into same tendency, equal tendency, and contrary tendency by the concept of set pair theory. The application of this model is empirically verified with Chinese enterprises. Through both static and dynamic evaluations of the knowledge management risk, this paper can effectively analyze risk development trend and provide decision support for the enterprise manager about the uncertain risks of knowledge management.
\end{abstract}

\section{Introduction}

Knowledge is one of the most important resources of an enterprise. Skillful knowledge management can bring significant benefits including labor productivity growth, an increase in quality of provided services, and also a strengthening of the competitive position of an enterprise [1]. Compared to other kinds of assets, knowledge has a unique nature in which the more it is used the more its value increases [2]. While benefiting from knowledge management, enterprises also face the uncertain risks from it. Knowledge management risk exists in the process of acquiring, integrating, and sharing knowledge, as well as in innovation and knowledge application, and it can bring loss to enterprise for some reasons. Some experts say that the failure ratio of knowledge management programs is between $50 \%$ and $70 \%$, which makes the evaluation and prevention of knowledge management risks especially important.

The research on risk management in academic world exists early, while the research on knowledge management risk appears later. Recently, the study on knowledge management risk has spread out. Ruggles in the research of factors influencing knowledge management application showed that uncoordinated organization culture is the biggest hindrance of knowledge management [3]. McBriara et al. studied the influence of knowledge management risk on knowledge management planning [4]. Elias and Wright pointed out that reasons behind knowledge management risks can be improper information policy, employee loss, and poor information transferability [5]. Perrott after combining the actual company situation proposed the top-down knowledge management system by establishing risk management committee including CEO, CFO, and risk management staff to monitor knowledge management risk [6]. Now, there are also lots of studies on knowledge management risk evaluation. Huang stated the contents of knowledge management risks and methods of how to identify and evaluate enterprise knowledge management risk from risk factors analysis, risk frequency, scope, gains, and losses estimation [7]. Zhang et al. put forward a multi-index complex evaluation method based on language evaluation for enterprise knowledge management risk [8]. Massingham has researched the cognitive constraints in knowledge risk management by using case study method [9]. Li and Zhang used extension method to evaluate 
enterprise information management risk [10]. Bamakan and Dehghanimohammadabadi propose a new quantitative risk analysis and assessment methodology, which is based on AHP and Monte Carlo simulation [11].

In general, knowledge management risk evaluation methods can be classified into qualitative method, quantitative method, and qualitative combining quantitative method, such as analytic hierarchy process, grey correlation method, and genetic algorithm. However, each method has certain limitations [12]. Qualitative method is fairly subjective and can be greatly affected by the evaluator; quantitative method describing the evaluation results through the objective data could make the result look too simple and cover up the role of other factors. The combination of qualitative and quantitative methods is the mainstream at this stage, which mainly includes the fuzzy comprehensive evaluation method and expert scoring method. These methods can evaluate the risk level of knowledge management; however, they cannot predict the risk development trend, which is exactly crucial information for enterprise managers. Since knowledge management risk refers to the uncertainties caused by variable factors during managing process and set pair theory is the mathematical method for uncertain issues with its core to describe uncertain issue by same-indefinite-contrary connection number and to predict system development trend [13], this paper introduced set pair analysis to evaluate knowledge management risk.

\section{Models and Methods}

2.1. Fundamentals of Set Pair Theory. Set pair analysis was first proposed by Chinese scholar Zhao in 1989 and it is a method to deal with fuzzy and uncertain problems [14]. Set pair is formed by two related sets, such as system and engineering, information and control, and production and sales. The basic idea is to do the same, indefinite, and the contrary analysis and quantitative description in the specific problem situation and then to get the connection degree expression based upon which one can study related issues of the system [15].

In set pair analysis, connection number [16] is an important concept and its general expression is

$$
\mu=\frac{S}{N}+\frac{F}{N} m+\frac{P}{N} n .
$$

In this expression, $N$ stands for all features of the set pair, $S$ stands for the same features, $F$ stands for the indefinite features, and $P$ stands for the contrary features. The connection among them is $N=S+P+F$. If we consider $a=S / N$, $b=F / N$, and $c=P / N$, then we can easily get this:

$$
\mu=a+b m+c n .
$$

According to expression (1), we know the relationship of $a, b$, and $c$ is $a+b+c=1$, with $a, b$, and $c$ belonging to $[0,1]$, $m \in[-1,1]$, and $n=-1, a$ being the same degree, $b$ the indefinite degree, and $c$ the contrary degree. The coefficient of " $a$ " is 1 , completely in the positive level. The coefficient of " $b$ " is $[-1,1]$, some in the positive level and some in the negative level. The coefficient of " $c$ " is -1 , completely in the negative level. If we use " $>$ " to express the location of $a, b$, and $c$, then we get $a>b>c$, with " $>$ " meaning "positive" [17].

2.2. Five-Element Connection Number. By the set pair analysis, we know connection number $\mu=a+b m+c n$ is a system in which $a, b, c$, and $j$ reflect the macro level, $i$ reflects the micro level, and $b i$ reflects both. So we can unfold it to express the hierarchy of the system, which is $\forall a, b, c \in[0,1], m \in[-1,1]$, and $n=-1$ and its general expression is

$$
\mu=a+b_{1} m_{1}+b_{2} m_{2}+\cdots+b_{n} m_{n}+c n .
$$

When $n=3$, we can get

$$
\begin{aligned}
\mu & =a+b_{1} m_{1}+b_{2} m_{2}+b_{3} m_{3}+c n, \\
\text { Or } \mu & =a+b i+c j+d k+e l .
\end{aligned}
$$

This expression is the so called five-element connection number, with $a$ being the same degree, $b$ being the indefinite same degree, $c$ being the indefinite center degree, $d$ being the indefinite contrary degree, and $e$ being the contrary degree. All of them are called connection degree, with $a, b, c, d, e \in$ $[0,1], a+b+c+d+e=1, i \in[0,1], j \in[0,0], k \in[-1,0]$, and $l=-1$. The relationship between $a, b, c, d$, and $e$ is $a>b>c>d>e$. In the information risk assessment, we can combine risk grade with the five-element connection number to further define the risk degree by using $a$ for "lowest risk," $b$ for "lower risk," $c$ for "medium risk," $d$ for "higher risk," and $e$ for "highest risk."

2.3. Evaluation Model for Risk Uncertainties. According to the fundamentals of set pair and five-element connection number, the influence factor of knowledge management risk sets is $U=\left(\mu_{1}, \mu_{2}, \ldots, \mu_{n}\right)$ and the weight matrix of influence factors is $W=\left(\omega_{1}, \omega_{2}, \ldots, \omega_{n}\right)$. Knowledge management risk evaluation of the people under survey for the influence factors can be divided into five classes, "lowest risk," "lower risk," "medium risk," "higher risk," and "highest risk." Combining the weights of affecting factors, we can get the following evaluation model [18]:

$$
\begin{aligned}
V= & \left(\omega_{1}, \omega_{2}, \ldots, \omega_{n}\right) \\
& \cdot\left\{\begin{array}{ccccc}
\mu_{11} & \mu_{12} & \mu_{13} & \mu_{14} & \mu_{15} \\
\mu_{21} & \mu_{22} & \mu_{23} & \mu_{24} & \mu_{25} \\
\vdots & \vdots & \vdots & \vdots & \vdots \\
\vdots & \vdots & \vdots & \vdots & \vdots \\
\mu_{n 1} & \mu_{n 2} & \mu_{n 3} & \mu_{n 4} & \mu_{n 5}
\end{array}\right\}\left(\begin{array}{c}
1 \\
i \\
j \\
k \\
l
\end{array}\right)=\sum_{t=1}^{n} \omega_{t} \mu_{t 1} \\
& +\sum_{t=1}^{n} \omega_{t} \mu_{t 2} i+\sum_{t=1}^{n} \omega_{t} \mu_{t 3} j+\sum_{t=1}^{n} \omega_{t} \mu_{t 4} k+\sum_{t=1}^{n} \omega_{t} \mu_{t 5} l .
\end{aligned}
$$


In this model, $E=\left(\begin{array}{lllll}1 & i & j & k & l\end{array}\right)^{T}$ is the coefficient matrix. According to set pair theory,

$$
\begin{aligned}
& a=\sum_{t=1}^{n} \omega_{t} a_{t 1} \text { reflects the "lowest risk," } \\
& b=\sum_{t=1}^{n} \omega_{t} u_{t 2} i \text { reflects the "lower risk," } \\
& c=\sum_{t=1}^{n} \omega_{t} u_{t 3} j \text { reflects the "medium risk," } \\
& d=\sum_{t=1}^{n} \omega_{t} u_{t 4} k \text { reflects the "higher risk," } \\
& e=\sum_{t=1}^{n} \omega_{t} u_{t 5} l \text { reflects the "highest risk." }
\end{aligned}
$$

\subsection{Risk Trend Analysis Method}

2.4.1. Partial Connection Number. Connection number can reflect the structure function of the system and the priorities of system levels. Partial connection number is the associate function of connection function [19] which can reflect the development trend of the system.

Let us define each partial connection number of fiveelement connection number [20].

First partial connection number is

$$
\partial \mu=\partial a+i \partial b+j \partial c+k \partial d,
$$

with $\partial a=a /(a+b), \partial b=b /(b+c), \partial c=c /(c+d)$, and $\partial d=$ $d /(d+e)$.

Second partial connection number is

$$
\partial^{2} \mu=\partial(\partial \mu)=\partial^{2} a+i \partial^{2} b+j \partial^{2} c,
$$

with $\partial^{2} a=\partial a /(\partial a+\partial b), \partial^{2} b=\partial b /(\partial b+\partial c), \partial^{2} c=\partial c /(\partial c+$ $\partial d)$.

Third partial connection number is

$$
\partial^{3} \mu=\partial\left(\partial^{2} \mu\right)=\partial^{3} a+i \partial^{3} b,
$$

with $\partial^{3} a=\partial^{2} a /\left(\partial^{2} a+\partial^{2} b\right) . \partial^{3} b=\partial^{2} b /\left(\partial^{2} b+\partial^{2} c\right)$.

Fourth partial connection number is

$$
\partial^{4} \mu=\partial\left(\partial^{3} \mu\right)=\partial^{4} a,
$$

with $\partial^{4} a=\partial^{3} a /\left(\partial^{3} a+\partial^{3} b\right)$.

From expression (7), we can tell $a$ in $\mu$ originates from $b$, and $\partial a=a /(a+b)$ reflects the development level in quantity; $b$ originates from $c$, and $\partial b=b /(b+c)$ reflects the development level in quantity; $c$ originates from $d$, and $\partial c=c /(c+d)$ reflects the development level in quantity; $d$ originates from $e$, and $\partial d=d /(d+e)$ reflects the development level in quantity. By the same token, we can get the meaning of other partial connection numbers.

2.4.2. Set Pair Tendency. Set pair tendency can reflect the same-indefinite-contrary degree of two sets [21]. In the fiveelement connection $\mu=a+b i+c j+d k+e l$, when $e \neq 0$, we call $a / e$ set pair tendency, or $\operatorname{shi}(H)=a / e$. Its specific meaning is as follows. When $a>e, a / e>1$, the two sets are in the same tendency. When $a<e$, a/e $<1$, the two sets are in the contrary tendency. When $a=e, a / e=1$, the two sets are in the equal tendency [22]. In the assessment of knowledge management risk, we can recognize "low risk" as the ideal reference set and get shi(risk) $=a / e$. Similarly, we can classify the knowledge management risk into three types: same tendency, contrary tendency, and equal tendency.

When the knowledge management risk is in the same tendency, it means that actual and ideal risk has the same trend and the actual risk is low. At this point, the managers should pay attention to the first partial connection number of each influence factor and invest more into influence factors in the contrary tendency to make it change to same tendency.

When the knowledge management risk is in the equal tendency, it means the actual risk is in the middle and the system risk is medium. At this point, managers should focus on the first partial connection number and prevent the risk from turning into contrary tendency.

When the information risk is in the contrary tendency, it means the actual risk is higher and the system risk is higher. At this point, managers should concentrate on every factor which is in contrary tendency and push them to change to equal tendency and then to same tendency.

\section{Empirical Analysis}

Due to the human uncertainties, knowledge itself and knowledge management environment, there are unavoidable risks in the process of using knowledge management methods to solve problems or in conducting knowledge management programs. Risks come along with profits, so it is of great importance to identify, evaluate, and manage risks for the enterprise when applying knowledge management. So far, there are lots of researches on the composition of knowledge management risk. Elias and Wright pointed out that reasons behind knowledge management risks can be improper information policy, employee loss, and poor information transferability [5]. Li and Zhang thought there are human resources risk, information risk, organization risk, technology risk, information process risk, and capital risk in knowledge management risk that enterprises face [10].

After combining the research results of scholars and the authors' investigation of manufacturing enterprises, this paper suggests five risks of knowledge management: human factor risk, information risk, organization risk, technology risk, and market risk. Meanwhile, the five risks can be measured by subordinate influencing factors. All these are shown as in Table 1 . Here we will explain the subordinate influencing factors. Employee loss means the probability that employees may leave the enterprise. Secret leakage is a risk that anyone in the enterprise may divulge a secret to the competitors, especially the intellectual property. Professional skill means the personal qualities and abilities of the employees. Information transform is the deviation between the explicit and tacit knowledge conversion. Information spill means there is a certain risk that, during the storage of knowledge, it may be stolen by hacker or spilled by internal staffs. Information transfer represents the risk during the process of transfer. Information property stands for the knowledge itself, which means the forms of knowledge. Strategy positioning is the positioning which the company sets for itself. Enterprise policy and enterprise capital stand 
TABLE 1: Five-element connection number table for influencing factors of evaluating knowledge management.

\begin{tabular}{|c|c|c|c|c|}
\hline \multirow{2}{*}{ Risks } & \multirow{2}{*}{ Risk factors } & \multirow{2}{*}{ Weight } & \multicolumn{2}{|c|}{ Five-element connection number } \\
\hline & & & Lowest, lower, medium, higher, highest & Tendency \\
\hline \multirow{4}{*}{ Human factor risk (0.18) } & Employee loss & 0.18 & $0.01+0.15 i+0.30 j+0.30 k+0.24 l$ & Contrary tendency \\
\hline & Secret leakage & 0.28 & $0.22+0.21 i+0.30 j+0.15 k+0.12 l$ & Same tendency \\
\hline & Professional skills & 0.54 & $0.15+0.19 i+0.20 j+0.21 k+0.25 l$ & Contrary tendency \\
\hline & Subtotal & 1.00 & $0.14+0.19 i+0.25 j+0.21 k+0.21 l$ & Contrary tendency \\
\hline \multirow{5}{*}{ Information risk (0.17) } & Information transform & 0.12 & $0.12+0.13 i+0.10 j+0.27 k+0.38 l$ & Contrary tendency \\
\hline & Information spill & 0.36 & $0.14+0.10 i+0.28 j+0.22 k+0.26 l$ & Contrary tendency \\
\hline & Information transfer & 0.34 & $0.27+0.30 i+0.20 j+0.13 k+0.10 l$ & Same tendency \\
\hline & Information property & 0.18 & $0.11+0.28 i+0.32 j+0.19 k+0.10 l$ & Same tendency \\
\hline & Subtotal & 1.00 & $0.18+0.20 i+0.24 j+0.19 k+0.19 l$ & Contrary tendency \\
\hline \multirow{4}{*}{ Organization risk $(0.20)$} & Strategy positioning & 0.44 & $0.12+0.19 i+0.48 j+0.11 k+0.10 l$ & Same tendency \\
\hline & Enterprise policy & 0.30 & $0.19+0.11 i+0.31 j+0.20 k+0.19 l$ & Equal tendency \\
\hline & Enterprise capital & 0.26 & $0.02+0.10 i+0.28 j+0.37 k+0.23 l$ & Contrary tendency \\
\hline & Subtotal & 1.00 & $0.12+0.14 i+0.38 j+0.20 k+0.16 l$ & Contrary tendency \\
\hline \multirow{3}{*}{ Technology risk (0.13) } & Management technique & 0.46 & $0.12+0.28 i+0.38 j+0.11 k+0.11 l$ & Same tendency \\
\hline & System usage & 0.54 & $0.42+0.25 i+0.21 j+0.08 k+0.04 l$ & Same tendency \\
\hline & Subtotal & 1.00 & $0.28+0.26 i+0.29 j+0.09 k+0.07 l$ & Same tendency \\
\hline \multirow{4}{*}{ Market risk (0.32) } & Customer satisfaction & 0.21 & $0.05+0.11 i+0.28 j+0.31 k+0.25 l$ & Contrary tendency \\
\hline & Time delay & 0.63 & $0.11+0.19 i+0.20 j+0.29 k+0.21 l$ & Contrary tendency \\
\hline & Outside change & 0.16 & $0.02+0.07 i+0.20 j+0.23 k+0.48 l$ & Contrary tendency \\
\hline & Subtotal & 1.00 & $0.08+0.15 i+0.22 j+0.28 k+0.26 l$ & Contrary tendency \\
\hline \multicolumn{2}{|c|}{ Total } & 1.00 & $0.14+0.18 i+0.27 j+0.21 k+0.20 l$ & Contrary tendency \\
\hline
\end{tabular}

The meaning of some risk factors:

Professional skills: the ability of staff is not enough to do the work.

Information transform: the deviation between the explicit and tacit knowledge conversion.

System usage: the employees reject the system or use it incorrectly.

Time delay: the speed of latest knowledge absorption is slow.

for the emphasis on knowledge management. Management technique is the method that managers take to control the risks. System usage means the employees reject the system or use it incorrectly. Time delay is the speed of latest knowledge absorption being slow, which could increase the risk of knowledge management. Outside change means the markets environments changes which could bring risks to the knowledge management.

When we compare all the risk factors to the relative standard, we can classify the risk into lowest risk, lower risk, medium risk, higher risk, and highest risk. In this way, we can build an evaluation model of knowledge management risk to analyze the influencing factors.

Weight of the influencing factors will be decided by integrative assigning method. The subjective assigning method by analytic hierarchy process (AHP) is suitable for subjects affected by many factors that are clearly distinctive, and the objective assigning method by entropy is to decide weight by decision matrix. After going through the subjective and objective assigning methods, the multiplicative method of integrative assigning is used to treat the two results and get the final reasonable weight for the risk evaluation factors. The detailed weight is shown in Table 1 . This survey takes manufacturing enterprise as the investigation subject and questionnaires are designed according to maximum difference. We did the survey by interviewing the MBA students who worked as managers in the manufacturing enterprises and let them fill in the questionnaire we designed. Among the 300 copies sent out to the managers in the manufacturing enterprises, 247 are back and 208 are valid. The empirical research includes 300 managers in 20 manufacturing enterprises. We have surveyed 8 automobile manufacturing enterprises and 6 electronic products manufacturing enterprises and we have handed out 120 questionnaires to the automobile manufacturing enterprises managers and 90 questionnaires to the electronic products manufacturing enterprises managers. On the other hand, we also have investigated 6 equipment manufacturing enterprises through the network and surveyed 90 managers of the enterprises via e-mail and 75 was the value which had been returned. The data and calculation results are shown in Tables 1, 2, and 3.

After analyzing the five-element connection number based on set pair theory, we can get below results for Table 1 data.

The five-element connection number for manufacturing enterprise is $0.14+0.18 i+0.27 j+0.21 k+0.20 l$, with set pair $\operatorname{shi}(H)=a / e=0.7$ in the contrary tendency, which means Chinese manufacturing managers today have high risk expectation on knowledge management process. Within it, human factor risk, information risk, organization risk, 


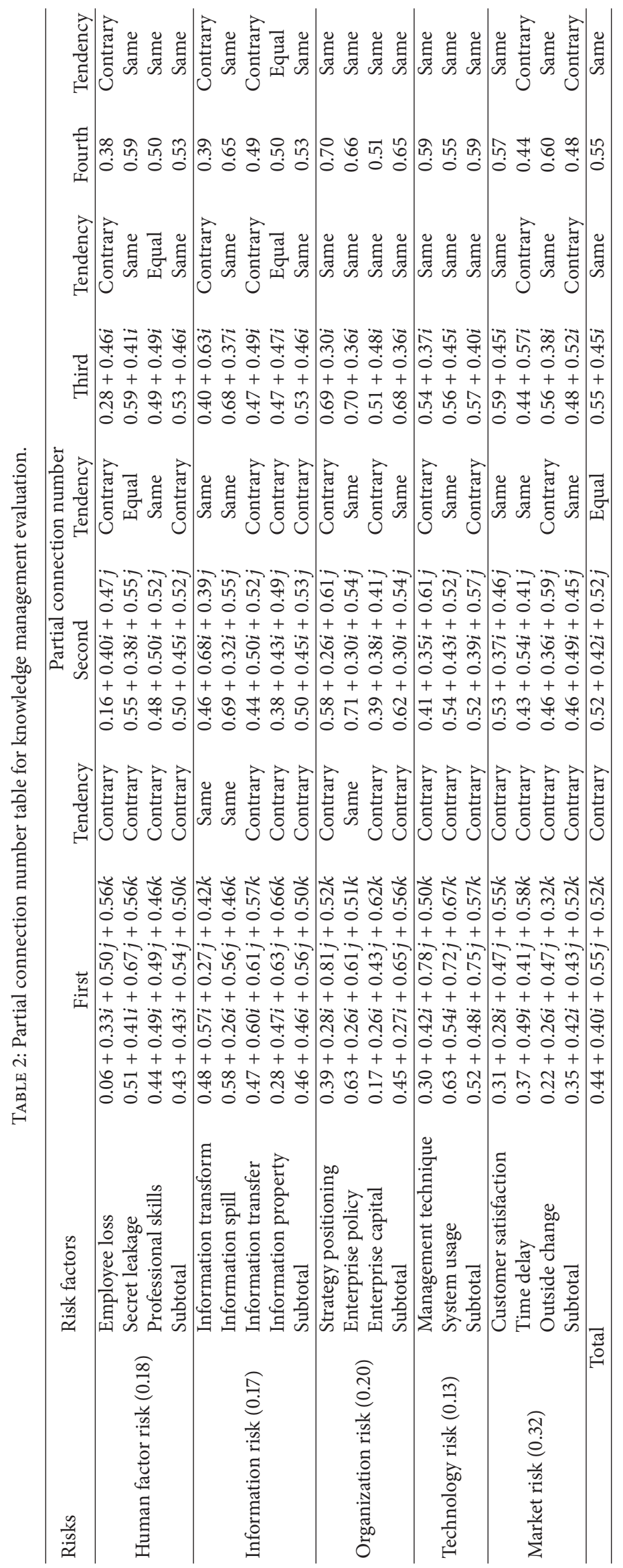


TABLE 3: Set pair variation table for knowledge management evaluation.

\begin{tabular}{|c|c|c|c|c|c|c|}
\hline Risks & Risk factors & $\begin{array}{c}\text { Five-element } \\
\text { connection number } \\
\text { set pair tendency }\end{array}$ & $\begin{array}{l}\text { First set pair } \\
\text { tendency }\end{array}$ & $\begin{array}{l}\text { Second set pair } \\
\text { tendency }\end{array}$ & $\begin{array}{l}\text { Third set pair } \\
\text { tendency }\end{array}$ & $\begin{array}{c}\text { Fourth set pair } \\
\text { tendency }\end{array}$ \\
\hline \multirow{4}{*}{ Human factor risk } & Employee loss & 0.04 & 0.11 & 0.33 & 0.62 & 0.38 \\
\hline & Secret leakage & 1.83 & 0.92 & 1.02 & 1.44 & 0.59 \\
\hline & Professional skills & 0.60 & 0.97 & 0.92 & 0.99 & 0.50 \\
\hline & Subtotal & 0.67 & 0.87 & 0.96 & 1.15 & 0.53 \\
\hline \multirow{5}{*}{ Information risk } & Information transform & 0.32 & 1.16 & 1.17 & 0.64 & 0.39 \\
\hline & Information spill & 0.54 & 1.27 & 1.25 & 1.86 & 0.65 \\
\hline & Information transfer & 2.70 & 0.84 & 0.85 & 0.96 & 0.49 \\
\hline & Information property & 1.10 & 0.43 & 0.77 & 1.01 & 0.50 \\
\hline & Subtotal & 0.94 & 0.93 & 0.95 & 1.13 & 0.53 \\
\hline \multirow{4}{*}{ Organization risk } & Strategy positioning & 1.20 & 0.74 & 0.95 & 2.32 & 0.70 \\
\hline & Enterprise policy & 1.00 & 1.24 & 1.30 & 1.96 & 0.66 \\
\hline & Enterprise capital & 0.09 & 0.27 & 0.94 & 1.05 & 0.51 \\
\hline & Subtotal & 0.75 & 0.78 & 1.15 & 1.89 & 0.65 \\
\hline \multirow{3}{*}{ Technology risk } & Management technique & 1.09 & 0.60 & 0.68 & 1.47 & 0.59 \\
\hline & System usage & 10.5 & 0.94 & 1.02 & 1.23 & 0.55 \\
\hline & Subtotal & 4.00 & 0.91 & 0.91 & 1.42 & 0.59 \\
\hline \multirow{4}{*}{ Market risk } & Customer satisfaction & 0.20 & 0.56 & 1.14 & 1.31 & 0.57 \\
\hline & Time delay & 0.52 & 0.63 & 1.04 & 0.77 & 0.44 \\
\hline & Outside change & 0.04 & 0.69 & 0.78 & 1.49 & 0.60 \\
\hline & Subtotal & 0.31 & 0.67 & 1.01 & 0.93 & 0.48 \\
\hline \multicolumn{2}{|c|}{ Total } & 0.70 & 0.84 & 1.01 & 1.23 & 0.55 \\
\hline
\end{tabular}

and market risk are in the contrary tendency, and only the technology tendency is in same tendency.

The five-element connection number for human factor risk is $0.14+0.19 i+0.25 j+0.21 k+0.21 l$ and, in the contrary tendency, indicates that human factor risk is high. In this type of risk, employee loss and professional skills are in the contrary tendency and secret leakage is in same tendency, suggesting that employee loss is serious and professional skills need further improvement, while secret leakage risk is low.

The five-element connection number for information risk is $0.18+0.20 i+0.24 j+0.19 k+0.19 l$ and is in the contrary tendency. The information transform and information spill are in the contrary tendency and the other two are in same tendency, which means that, in manufacturing enterprise, explicit information and implicit information transform can have deviation easily and information is easy to be imitated or gained by competitors.

The five-element connection number for organization risk is $0.12+0.14 i+0.38 j+0.20 k+0.16 l$ and is in the contrary tendency, with strategy positioning and enterprise policy in same tendency and equal tendency, respectively, and enterprise capital in contrary tendency, indicating that, in manufacturing enterprise capital, usage efficiency is not high and input and output measurements are not correct.
The five-element connection number for technology risk is $0.28+0.26 i+0.29 j+0.09 k+0.07 l$ and is in same tendency, with knowledge management technology and system usage in same tendency, suggesting small risk.

The five-element connection number for market risk is $0.08+0.15 i+0.22 j+0.28 k+0.26 l$ and is in contrary risk, with customer satisfaction, time delay, and outer environment all in contrary tendency, which means that market changes fast and customers have higher product requirement; however, the product design and production of today's manufacturing enterprise can hardly follow the consumer's taste change.

According to set pair theory, data in Table 2 can be analyzed as shown below.

The first partial connection number for knowledge management of manufacturing enterprise is $0.44+0.40 i+0.55 j+$ $0.52 k$ and is in contrary tendency, showing rising risk in knowledge management of today's redt, the first partial connection number of human factor, information, organization, technology, and market are all in the contrary tendency, suggesting all the risks are increasing. Among the 15 risk factors, only information transform, information spill, and enterprise policy are in the same tendency, and the other 12 factors are in the contrary tendency. Managers should pay attention to all the risk factors of the partial connection number that are in contrary tendency and take actions to prevent further increasing of the risks. 
The second partial connection number for knowledge management of manufacturing enterprise is $0.52+0.42 i+$ $0.52 j$ and is in equal tendency, while the third partial connection number is $0.55+0.45 i$, in equal tendency, and the fourth is 0.55 , bigger than 0.5 , and is in equal tendency.

Based on set pair theory, we can draw the following conclusions for data in Table 3.

The set pair tendency of the whole five-element connection number is 0.70 , less than 1 , and is in the contrary tendency. The set pair tendencies of the five risks are 0.67, $0.94,0.75,4.00$, and 0.31 , respectively, with four in contrary tendency, accounting for $80 \%$. Only technology risk is in same tendency, which means the technology risk is the lowest in the five factors, because in the survey, the managers all think the system usage is the progress which could be controlled easily and the system usually should be trusted. Market risk set pair tendency is the smallest, meaning market risk is the highest. In human factor risk, employee loss is the highest risk, while in information risk, information transform is the highest risk, and in organization risk, enterprise capital is the highest risk, and in market risk, outside environment change is the highest risk. Enterprise managers should pay great attention to the four risk factors and take measures for prevention. From the set pair tendency of the first partial connection number, information transform, information spill, and enterprise policy among the 15 risk factors are bigger than 1 for set pair tendency and are in the same tendency with others in contrary tendency. This means today's manufacturing enterprise faces increasing knowledge management risk.

The set pair tendency of all the manufacturing enterprise is $0.7,0.84,1.01,1.23$, and 0.55 , respectively, indicating that the risks are moving towards same tendency with big step, which requires the managers to take actions to facilitate the change in order to reduce risk.

\section{Conclusions}

Chinese manufacturing enterprise now faces great and rising risk in knowledge management, which pushes managers to take measures to reduce the risk factors in contrary tendency. For instance, in human factor risk, employee welfare should be increased to lower the employee loss rate, and employee training or hiring employees with better professional skills is needed to improve the overall employee professional skill; leaders should communicate with employees to find out the employees' needs, enhance organizational cohesion, and avoid the employees' departure due to dissatisfaction with the enterprise. In organization risk, the managers should build a positive and open enterprise culture to promote employees and enterprises set up the shared values and vision. In addition, open enterprise interior environment atmosphere can not only make employees more likely to accept new things and effectively avoid repellency of knowledge management system, but also accelerate the speed of knowledge innovation. In technology risk, enterprises should strengthen staff training and enhance the staff's comprehensive quality and professional skills. In market risk, the managers should pay attention to the competitors. It can help enterprise understand the market changes; we can get some knowledge of the competitors when they collect the market information.

Set pair analysis is a mathematical tool to study uncertain system. Using the connection number concept of set pair theory to evaluate knowledge management risk can get the static tendency of the risks, as well as the prediction of their development trend, realizing the way of combining static and dynamic and quality and quantity for the evaluation. From the above analysis, we can see that the connection number of the risks can be in same, equal, and contrary tendency, moving forward in waving momentum. This requires the manager to make preparations to identify the risk development tendency and take measurements in human, capital, and other aspects to lower risks. Set pair analysis can also be supplied to research information risk evaluation, such as the information diffusion system. At the same time, it can be used to evaluate the construction program risk, such as the natural gas pipeline construction.

When analyzing the set pair tendency, this paper did not differentiate the weakness and strength of each tendency, which needs further discussion in future research.

\section{Competing Interests}

The authors declare that there are no competing interests regarding the publication of this paper.

\section{Acknowledgments}

This work was supported by the research fund of Social Science Fund Project of Heilongjiang Province (13B010) and Natural Science Fund Project of Heilongjiang Province (G201114).

\section{References}

[1] B. Hoła, M. Sawicki, and M. Skibniewski, "An IT model of a Knowledge Map which supports management in small and medium-sized companies using selected Polish construction enterprises as an example," Journal of Civil Engineering and Management, vol. 21, no. 8, pp. 1014-1026, 2015.

[2] M. Jafari, J. Rezaeenour, M. M. Mazdeh, and A. Hooshmandi, "Development and evaluation of a knowledge risk management model for project-based organizations: a multi-stage study," Management Decision, vol. 49, no. 3, pp. 309-329, 2011.

[3] R. Ruggles, "The state of the notion: knowledge management in practice," California Management Review, vol. 40, no. 3, pp. 80-89, 1998.

[4] I. McBriara, C. Smith, G. Bainc et al., "Risk, gap and strength: key concepts in knowledge management," Information-Based Systems, vol. 16, no. 1, pp. 29-36, 2003.

[5] N. Elias and A. Wright, "Using knowledge management systems to manage knowledge resource risks," in Advances in Management Accounting, vol. 15, pp. 195-227, 2006.

[6] B. E. Perrott, "A strategic risk approach to knowledge management," Business Horizons, vol. 50, no. 6, pp. 523-533, 2007.

[7] L. Huang, "Identification of enterprise knowledge management," Enterprise Economy, no. 7, pp. 68-70, 2002. 
[8] X. Zhang, Z. Fan, and Y. Sun, "Analysis and evaluation of enterprise knowledge management risk," Journal of Northeastern University (Natural Science), vol. 24, no. 9, pp. 881-884, 2003.

[9] P. Massingham, "Knowledge risk management: a framework," Journal of Knowledge Management, vol. 14, no. 3, pp. 464-485, 2010.

[10] W. Li and Q. Zhang, "Enterprise knowledge management risk evaluation based on extension method," Journal of Intelligence, vol. 30, no. 12, pp. 129-134, 2011.

[11] S. M. H. Bamakan and M. Dehghanimohammadabadi, "A Weighted monte carlo simulation approach to risk assessment of information security management system," International Journal of Enterprise Information Systems, vol. 11, no. 4, pp. 6378, 2015.

[12] G. Han and N. Yu, "Application of improved OWA operator and intuitionistic fuzzy sets in decision-making of jack-up drilling platform design scheme," Journal of Chemical and Pharmaceutical Research, vol. 6, no. 6, pp. 1640-1646, 2014.

[13] K. Zhao, Set Pair Analysis and Its Preliminary Application, Zhejiang Science \& Technology Press, 2000.

[14] K. Zhao, "Set pair and set pair analysis: a new concept and new system analysis method," in Proceedings of the National System Theory and Regional Planning Conference, pp. 87-91, 1989.

[15] Y. Jiang and C. Xu, "Set pair analysis theory and its application status," Computer Science, vol. 33, no. 1, pp. 205-209, 2006.

[16] K. Zhao and H. Cao, The Research and Application of Set Pair Analysis and Shell Theory, China Meteorological Press, 2002.

[17] X. Wang, "Four-element connection number system tendency value ordering and application with 4 and 6 as the connection number," Mathematics in Practice and Theory, vol. 34, no. 7, pp. 107-112, 2004.

[18] X. Zhou and J. Zhang, "Risk evaluating method and application based on five-element connection number," System EngineeringTheory \& Practice, vol. 33, no. 8, pp. 2069-2176, 2013.

[19] K. Zhao, "Partial connection number," in Proceedings of the Progress of Artificial Intelligence in China, pp. 884-885, Beijing University of Posts and Telecommunications Press, 2005.

[20] T. Wu, "Application of five-element connection number in students score development trend," Mathematics in Practice and Theory, vol. 39, no. 5, pp. 53-59, 2009.

[21] D. Li, K. Xu, X. Zhang, and X. Dai, "Research and application of set pair tendency by set pair analysis," Industrial Safety and Environmental Protection, vol. 35, no. 9, pp. 8-9, 2009.

[22] R. L. Guo and K. Q. Zhao, "Study on the uncertainty trend and its tendency grades in similarity-difference connection degree," in Proceedings of Progress of Artificial Intelligence in China, pp. 1423-1426, Beijing University of Posts and Telecommunications Press, 2005. 

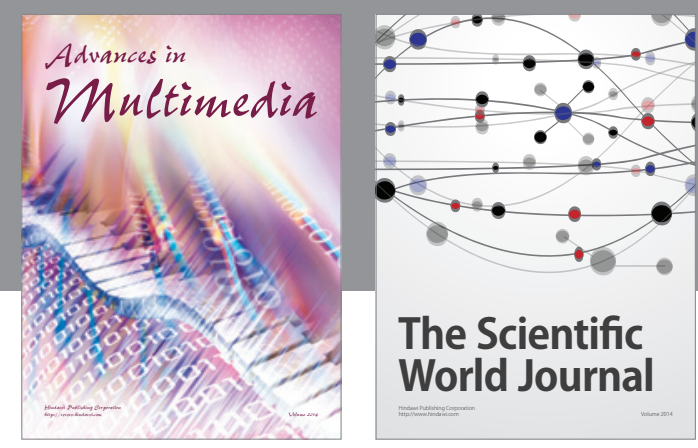

The Scientific World Journal
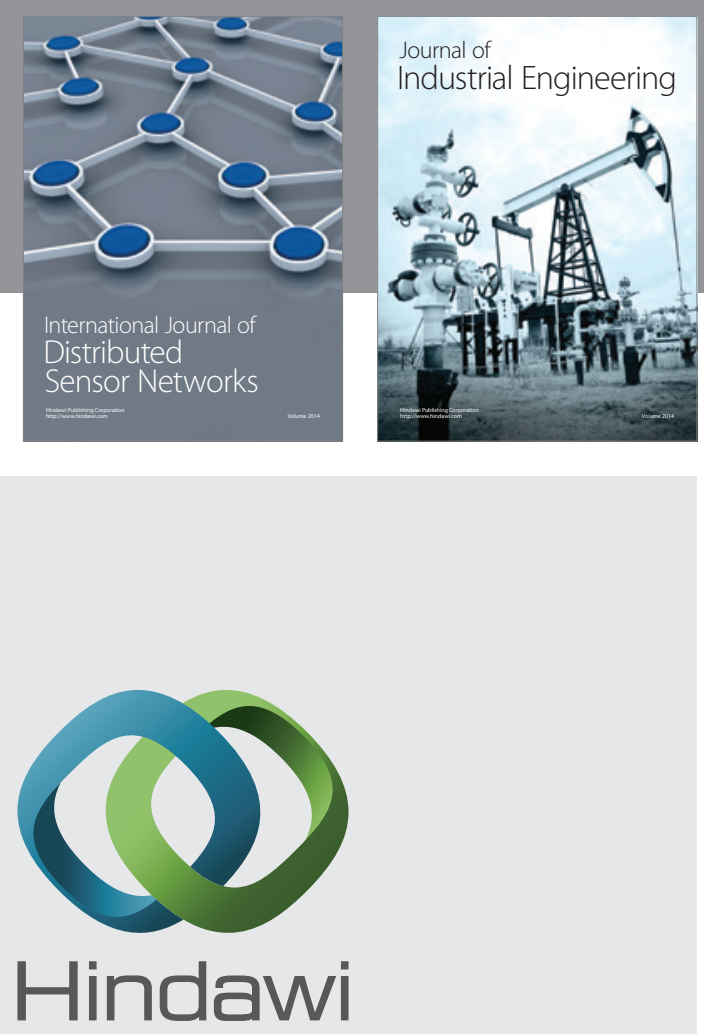

Submit your manuscripts at

http://www.hindawi.com

\section{Computer Networks} and Communications
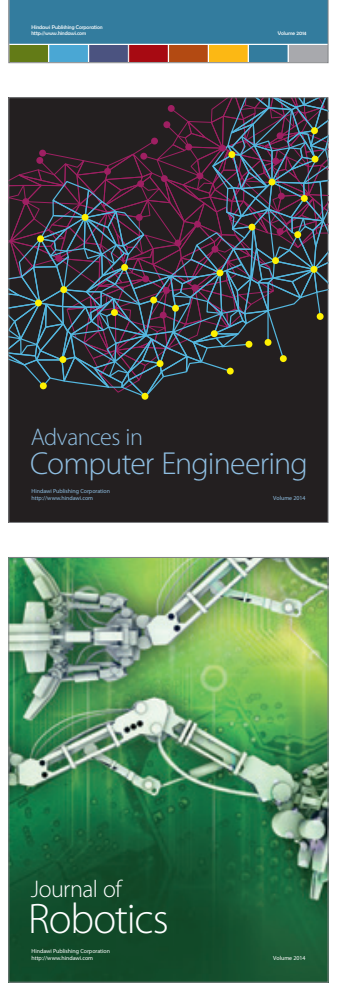
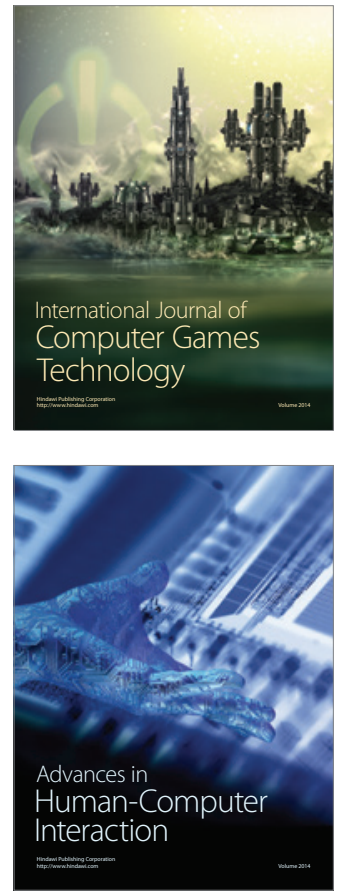
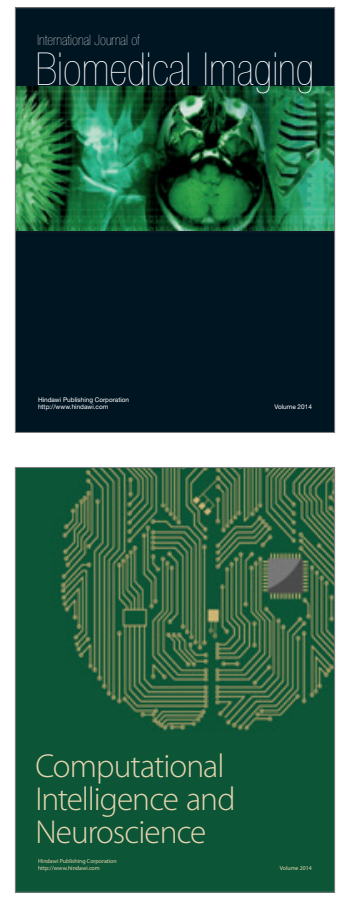
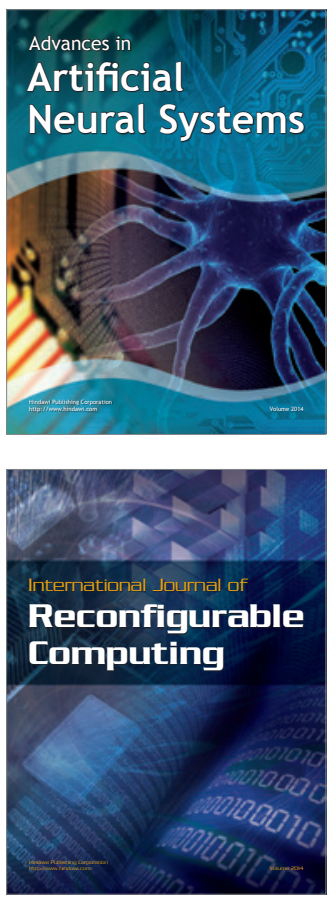
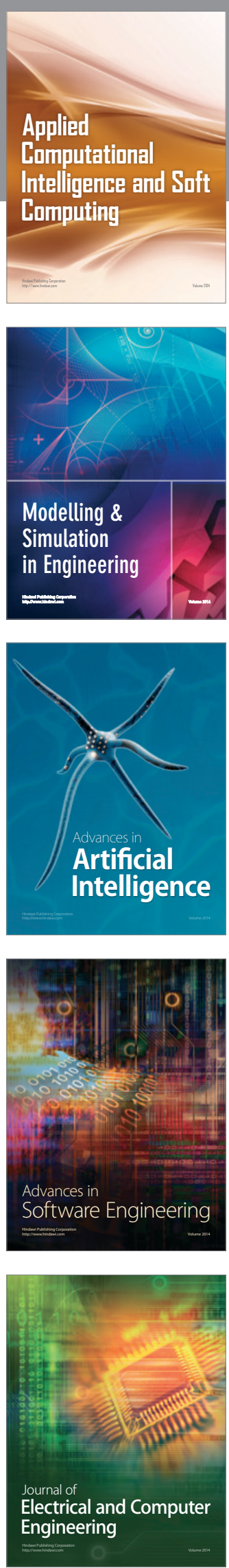